\title{
Dissection of experimental asthma with DNA microarray analysis identifies arginase in asthma pathogenesis
}

\author{
Nives Zimmermann, ${ }^{1}$ Nina E. King, ${ }^{1}$ Johanne Laporte, ${ }^{2}$ Ming Yang, ${ }^{3}$ Anil Mishra, ${ }^{1}$ \\ Sam M. Pope, ${ }^{1}$ Emily E. Muntel, ${ }^{1}$ David P. Witte, ${ }^{4}$ Anthony A. Pegg, ${ }^{5}$ Paul S. Foster, ${ }^{3}$ \\ Qutayba Hamid, ${ }^{2}$ and Marc E. Rothenberg ${ }^{1}$ \\ ${ }^{1}$ Division of Allergy and Immunology, Department of Pediatrics, Cincinnati Children's Hospital Medical Center, \\ Cincinnati, Ohio, USA \\ ${ }^{2}$ Meakins-Christie Laboratories and Montreal Chest Institute, McGill University, Montreal, Canada \\ ${ }^{3}$ Division of Molecular Biosciences, John Curtin School of Medical Research, Australian National University, \\ Canberra, Australia \\ ${ }^{4}$ Division of Pathology, Department of Pediatrics, Cincinnati Children's Hospital Medical Center, Cincinnati, Ohio, USA \\ ${ }^{5}$ Department of Cellular and Molecular Physiology, Pennsylvania State University College of Medicine, Hershey, \\ Pennsylvania, USA
}

\begin{abstract}
Asthma is on the rise despite intense, ongoing research underscoring the need for new scientific inquiry. In an effort to provide unbiased insight into disease pathogenesis, we took an approach involving expression profiling of lung tissue from mice with experimental asthma. Employing asthma models induced by different allergens and protocols, we identified $6.5 \%$ of the tested genome whose expression was altered in an asthmatic lung. Notably, two phenotypically similar models of experimental asthma were shown to have distinct transcript profiles. Genes related to metabolism of basic amino acids, specifically the cationic amino acid transporter 2 , arginase I, and arginase II, were particularly prominent among the asthma signature genes. In situ hybridization demonstrated marked staining of arginase I, predominantly in submucosal inflammatory lesions. Arginase activity was increased in allergen-challenged lungs, as demonstrated by increased enzyme activity, and increased levels of putrescine, a downstream product. Lung arginase activity and mRNA expression were strongly induced by IL- 4 and IL-13, and were differentially dependent on signal transducer and activator of transcription 6. Analysis of patients with asthma supported the importance of this pathway in human disease. Based on the ability of arginase to regulate generation of NO, polyamines, and collagen, these results provide a basis for pharmacologically targeting arginine metabolism in allergic disorders.
\end{abstract}

J. Clin. Invest. 111:1863-1874 (2003). doi:10.1172/JCI200317912.

\section{Introduction}

Despite intense ongoing asthma research, there is currently an epidemic of this disease in the western world and the incidence is on the rise $(1,2)$. Experimentation in the asthma field has largely focused on analysis of the cellular and molecular events induced by allergen exposure in sensitized animals (primarily mice) and humans. These studies have identified elevated production of

Received for publication January 20, 2003, and accepted in revised form April 1, 2003.

Address correspondence to: Marc Rothenberg, Division of Allergy and Immunology, Cincinnati Children's Hospital Medical Center, 3333 Burnet Avenue, Cincinnati, Ohio 45229, USA. Phone: (513) 636-7210; Fax: (513) 636-3310;

E-mail: Rothenberg@cchmc.org.

Nives Zimmermann and Nina E. King contributed equally to this work.

Conflict of interest: The authors have declared that no conflict of interest exists.

Nonstandard abbreviations used: cationic amino acid transporter (CAT); signal transducer and activator of transcription (STAT); argininosuccinate lyase (AL); argininosuccinate synthetase (AS); ornithine decarboxylase (ODC); ornithine aminotransferase (OAT).
IgE, mucus hypersecretion, airway obstruction, inflammation, and enhanced bronchial reactivity to spasmogens in the asthmatic response (3-5). Clinical and experimental investigations have demonstrated a strong correlation between the presence of $\mathrm{CD}^{+} \mathrm{Th} 2$ cells and disease severity, suggesting an integral role for these cells in the pathophysiology of asthma (6). Th2 cells are thought to induce asthma through the secretion of an array of cytokines (IL-4, $-5,-6,-9-10,-13$, and -25$)$ that activate inflammatory and residential effector pathways both directly and indirectly $(7,8)$. In particular, IL-4 and IL-13 are produced at elevated levels in the asthmatic lung and are thought to be central regulators of many of the hallmark features of disease $(9,10)$. Although these studies have provided the rationale for the development of multiple therapeutic agents that interfere with specific inflammatory pathways (11), the development of the asthma phenotype is likely to be related to the complex interplay of a large number of additional genes and their polymorphic variants. Accordingly, we aimed to identify new genes involved in the pathogenesis of experimental asthma with the use of an empirical 
approach that uses DNA microarray analysis of whole lung RNA. By using two phenotypically similar models of experimental asthma induced by independent regimes, we report distinct transcript profiles associated with each model. Among the asthma signature genes, we found concerted overexpression of the genes encoding for molecules involved in uptake and metabolism of basic amino acids (e.g., arginine), specifically, arginine metabolism by arginase. We chose to focus on these genes because intracellular arginine is a regulator of diverse pathways, including production of $\mathrm{NO}$, polyamines, and proline; these molecules regulate critical processes associated with asthma, including airway tone, cell hyperplasia, and collagen deposition, respectively $(12,13)$. Our results demonstrate specific regulation of arginase by diverse allergens, IL-4, and IL-13. In addition, we demonstrate overproduction of an arginase downstream product in the asthmatic lung. Finally, we provide evidence that this pathway is operational in human asthma.

\section{Methods}

Experimental asthma induction. Balb/c mice were obtained from the National Cancer Institute (Frederick, Maryland, USA) and signal transducer and activator of transcription 6-deficient (STAT6-deficient) mice $(\mathrm{Balb} / \mathrm{c})$ were obtained from Jackson Laboratory (Bar Harbor, Maine, USA); all mice were housed under specific pathogen-free conditions. Asthma models were induced by intraperitoneal injection with $100 \mu \mathrm{g}$ OVA (grade V, Sigma-Aldrich, St. Louis, Missouri, USA) and $1 \mathrm{mg}$ aluminum hydroxide (alum) as adjuvant twice, followed by two $50 \mu \mathrm{g}$ OVA or saline intranasal challenges 3 days apart, starting a least 10 days after the second sensitization, as previously described (14). The level of LPS in OVA was less than $2 \mathrm{pg} / \mathrm{ml}$ as detected by the Limulus assay. Mice were killed 3 or 18 hours after the first or second challenge. Aspergillus fumigatus antigen-associated asthma was induced by challenging mice intranasally three times a week for 3 weeks, as described (14-16). In brief, mice were lightly anesthetized with isofluorane inhalation and $100 \mu \mathrm{g}(50$ $\mu \mathrm{l})$ of A. fumigatus extract (Bayer Pharmaceuticals, Spokane, Washington, USA) or $50 \mu$ l of normal saline solution alone was applied to the nasal cavity by using a micropipette with the mouse held in the supine position. After instillation, mice were held upright until alert. Mice were killed 18 hours after the last challenge. This protocol induces a similar phenotype to the OVA model (characterized by eosinophilia, Th2 responses, eotaxin- 1 and eotaxin- 2 induction, and airway hyperreactivity) (refs. 14-17, and data not shown).

Preparation of RNA and microarray bybridization. RNA was extracted by using the Trizol reagent as per the manufacturer's instructions. After Trizol purification, RNA was repurified with phenol-chloroform extraction and ethanol precipitation. Microarray hybridization was performed by the Affymetrix Gene Chip Core facility at Cincinnati Children's Hospital Medical Center. Briefly,
RNA quality was first assessed by using the Agilent bioanalyzer (Agilent Technologies, Palo Alto, California, USA) and only those samples with $28 \mathrm{~S} / 18 \mathrm{~S}$ ratios between 1.3 and 2 were subsequently used. RNA was converted to cDNA with Superscript choice for cDNA synthesis (Invitrogen, Carlsbad, California, USA) and subsequently converted to biotinylated cRNA with Enzo High Yield RNA Transcript labeling kit (Enzo Diagnostics, Farmingdale, New York, USA). After hybridization to the murine U74Av2 GeneChip (Affymetrix, Santa Clara, California, USA), the gene chips were automatically washed and stained with streptavidin-phycoerythrin by using a fluidics system. The chips were scanned with a Hewlett-Packard GeneArray Scanner (HewlettPackard, Palo Alto, California, USA). This analysis was performed with one mouse per chip $(n=3$ or more for each allergen challenge condition and $n=2$ or more for each saline challenge condition). A further description of the methodology, according to MIAME (Minimum Information About a Microarray Experiment) guidelines (www.mged.org/Workgroups/MIAME/miame.html) is provided in supplementary information (http://www.jci. org/cgi/content/full/111/12/1863/DC1).

Microarray data analysis. From data image files, gene transcript levels were determined with the use of algorithms in the Microarray Analysis Suite software (Affymetrix). Global scaling was performed to compare genes from chip to chip; thus, each chip was normalized to an arbitrary value (1500). Each gene is typically represented by a probe set of 16 to 20 probe pairs. Each probe pair consists of a perfect match oligonucleotide and a mismatch oligonucleotide that contains a onebase mismatch at a central position. Two measures of gene expression were used, absolute call and average difference. Absolute call is a qualitative measure in which each gene is assigned a call of present, marginal, or absent, based on the hybridization of the RNA to the probe set. Average difference is a quantitative measure of the level of gene expression, calculated by taking the difference between mismatch and perfect match of every probe pair and averaging the differences over the entire probe set. Differences between saline- and allergen-treated mice were also determined with the use of the GeneSpring software (Silicon Genetics, Redwood City, California, USA). Data were normalized to the average of the saline-treated mice. Gene lists were created that contained genes with $P<0.05$ and greater than twofold change by log transformation of average difference values (by using genes that received a present call, which was based on the hybridization signal). Excel files (Microsoft, Redmond, Washington, USA) that include average differences and absolute call values for all 12,422 genes in the 11 analyzed DNA chips are presented in supplementary Table 1 (http://www.jci. org/cgi/content/full/111/12/1863/DC1).

Northern blot and RT-PCR analysis. RNA was extracted from the lungs of wild-type Balb/c mice, IL-4 Clara cell 10 lung transgenic mice (18) containing wild-type or deleted copies of the gene for STAT6 (all in the Balb/c 
background) (19), and from the lungs of mice treated with intranasal or intratracheal saline or recombinant murine IL-13 (obtained from Debra Donaldson, Wyeth Research, Madison, New Jersey, USA), as previously reported $(17,20,21)$. The cDNA probes, generated by PCR or from commercially available vectors (Image Consortium obtained from American Tissue Culture Collection, Rockville, Maryland, USA, or Incyte Genomics, Palo Alto, California, USA), were sequence confirmed, radiolabeled with ${ }^{32} \mathrm{P}$, and hybridized by using standard conditions. RT-PCR, which used standard procedures with gene specific primers, was performed by using lung cDNA as a template.

In situ bybridization of mouse lung. In situ hybridization was performed as described (22). In brief, murine arginase I cDNA in plasmid PCMV-SPORT6 (Incyte Genomics, St. Louis, Missouri, USA) was linearized by EcoRI or Not I digestion, and antisense and sense RNA probes, respectively, were generated by T7 and SP6 RNA polymerase (Riboprobe Gemini Core System II transcription kit; Promega, Madison, Wisconsin, USA). The radiolabeled $\left(\alpha \mathrm{S}^{35}\right.$-UTP) probes were hybridized and washed under high-stringency conditions.

Immunohistochemistry. Slides from saline- and OVA-challenged lung tissue were stained with the anti-arginase-I antibody (affinity-purified RG-1, kindly provided by Sidney Morris, University of Pittsburgh School of Medicine) (23) at $3.5 \mu \mathrm{g} / \mathrm{ml}$. After staining with a biotinylated antichicken secondary antibody at $15 \mu \mathrm{g} / \mathrm{ml}$ (Vector Laboratories Inc., Burlingame, California, USA) and streptavidin-Vectastain Elite ABC-peroxidase reagents (Vector Laboratories Inc.), slides were subsequently developed with diaminobenzidine (DAB) (Vector Laboratories Inc.). Anti-Mac-3 staining $(10 \mu \mathrm{g} / \mathrm{ml}$; BD Pharmingen, San Diego, California, USA) was performed with the Vectastain Elite ABC-Peroxidase Rat IgG kit for detection, according to the manufacturer's instructions. As negative controls, tissues were stained without primary antibody. Arginase activity. Arginase activity was measured by using the blood urea nitrogen reagent (Sigma-Aldrich, St. Louis, Missouri, USA) according to established techniques (23-25). In some experiments, arginase activity was measured in the J774A.1 macrophage cell line (ATCC), plated at $0.25 \times 10^{6} / \mathrm{ml}$ and stimulated with $0.5-50 \mathrm{ng} / \mathrm{ml} \mathrm{IL}-13$ for 18 hours. Arginase activity was measured in cell lysates, as described above.

Putrescine levels. After acid extraction, putrescine content was determined by ion-pair reverse-phase HPLC, by using methods previously described (26).

Analysis of buman lung samples. Fiberoptic bronchoscopy and endobronchial biopsy of the subsegmental bronchus of allergic asthmatics and healthy controls after their informed consent was conducted, by using methods previously reported $(27,28)$. Asthmatic patients (age range $27-44$ years, $75 \%$ male) were all atopic individuals requiring intermittent $\beta 2$-agonist therapy (forced expiratory volume in $1 \mathrm{~s}$ [FEV1] values of $78 \%-90 \%$ normal), not requiring inhaled glucocorticoids for at least 4 weeks, free of respiratory infections for at least 8 weeks, and not on immunotherapy for at least the last 4 years. Healthy, gender-matched controls (age range 29-44) were nonatopic and had FEV1 values greater than $95 \%$ normal. Bronchoalveolar lavage cells after cytocentrifugation (after methanol/acetone fixation) were immunohistochemically stained with monoclonal mouse IgG1 antihuman arginase I (BD Biosciences, Lexington, Kentucky, USA) by using 1/100 dilution. The slides were developed in Fast Red (SigmaAldrich) in the presence of levamisole, as described previously (29). For negative control preparations, the primary antibody was replaced by saline solution or nonimmune mouse IgG1. A minimum of 1000 cells on blindly coded cytospin slides were scored for the number of positive cells, expressed as a percentage of total cells. In situ hybridization of lung samples from asthmatic and control individuals was performed with radiolabeled $\left[\alpha S^{35}\right.$-UTP] sense and antisense probes hybridized and washed under high-stringency conditions, as previously described (28).

Statistical analysis. The significance of differences between the means of experimental groups was analyzed with Student unpaired $t$ test. Values were reported as the mean $\pm S D$. Differences in mean values were considered significant if $P<0.05$.

\section{Results}

DNA microarray analysis identifies a subset of the genome involved in asthma pathogenesis. We were first interested in reproducibly and accurately identifying genes that were differentially expressed in a well-established model of asthma. To accomplish this aim, mice were intraperitoneally sensitized with the allergen OVA in the presence of the adjuvant alum and subsequently challenged intranasally with OVA or control saline on two occasions separated by 3 days. Eighteen hours after the last allergen challenge, one lobe of the lung was subjected to histologic analysis and the remainder of the lung was used for RNA analysis. As expected, histologic analysis revealed that the allergen-challenged mice had a marked eosinophil-rich inflammatory response, as previously reported (30). To verify the presence of allergen-induced mRNA transcripts, the RNA was subjected to Northern blot analysis to analyze the induction of the chemokine eotaxin-1, which has previously been shown to be significantly induced by allergen challenge (30). The finding that the allergen-challenged lungs had abundant eotaxin-1 mRNA levels, whereas saline-treated mice had very low levels, validated the experimental induction protocol (Figure 1a). We next subjected the RNA to microarray analysis by using the Affymetrix chip U74Av2 that contains oligonucleotide probe sets representing 12,422 genetic elements, the largest collection of characterized mouse genes commercially available. Comparison of the two saline-challenged mice to each other and comparison of the two allergen-challenged mice to each other revealed $1 \%$ or less of the genes changing more than twofold. Analysis of present genes in a scatter plot revealed relatively few points outside of 
a
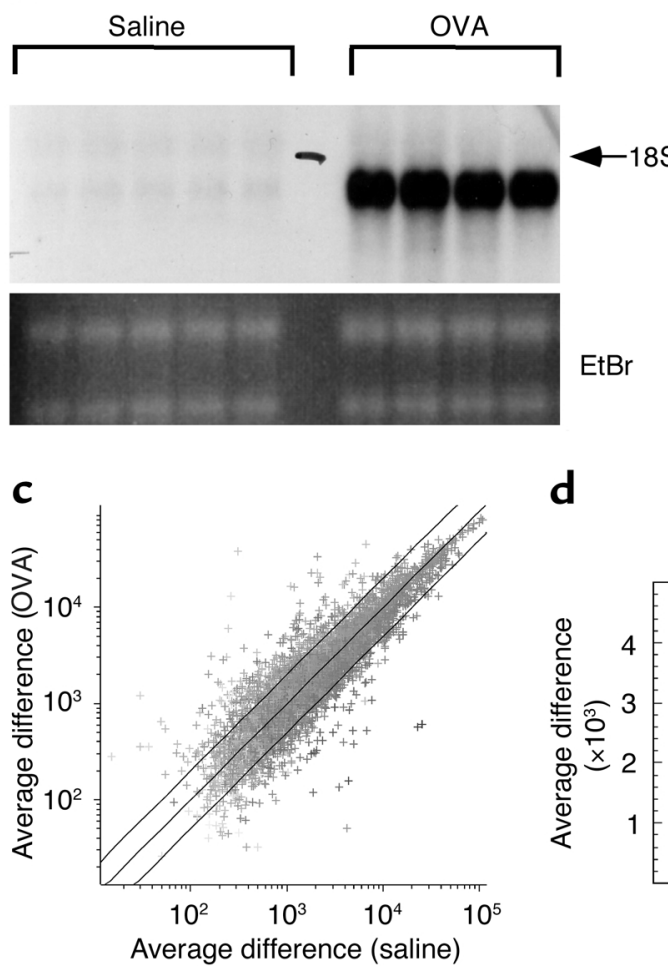

b

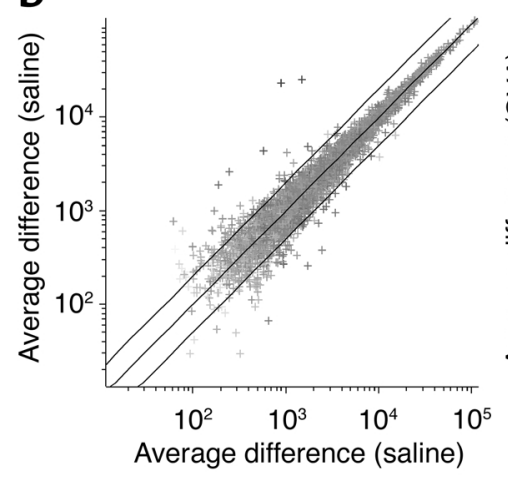

Average difference (saline)

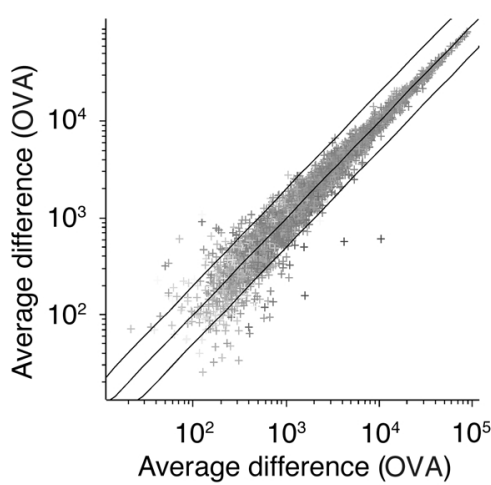

e

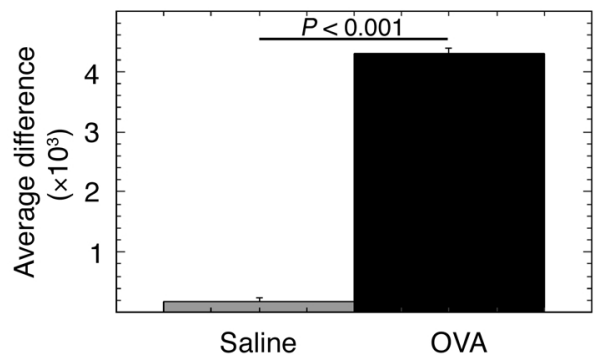

OVA Aspergillus (496 genes) (527 genes)

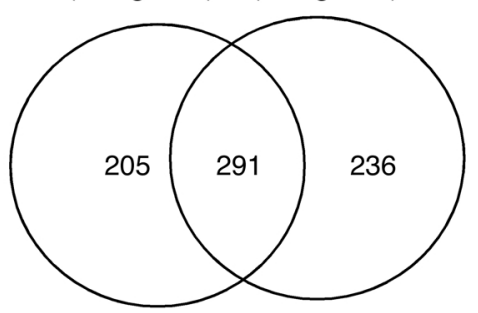

\section{Figure 1}

Microarray analysis of experimental asthma. In a, the induction of eotaxin-1 in allergen-challenged mice as measured by Northern blot analysis is shown. Total RNA $(10 \mu \mathrm{g})$ was electrophoresed, transferred, and hybridized with a radiolabeled eotaxin-1 cDNA probe. The location of 18S RNA is shown. Each lane represents a separate mouse. Ethidium bromide (EtBr) staining of the RNA gel is also shown. In $\mathbf{b}$, scatter plots of the average difference of present genes in two representative saline-challenged (left) or OVA-challenged (right) samples are shown. In $\mathbf{c}$, the average difference of present genes in a representative saline-treated sample compared with a representative OVA-treated sample is shown. In d, quantitative analysis of the eotaxin- 1 signal for saline- and OVA-treated mice is shown $(n=3$ mice each). Error bars represent the SD. In e, the number of genes increased in experimental asthma induced with OVA or A. fumigatus antigen is depicted in a Venn diagram. Data are derived from statistically significant bioinformatic analysis as described in the Methods section. Data are available in supplementary Tables 1-4 (http://www.jci.org/cgi/content/full/111/12/1863/DC1).

the twofold boundaries (Figure 1b). In contrast, pairwise comparison of allergen-challenged mice to salinechallenged mice revealed a greater than twofold change in $6.5 \% \pm 0.8 \%$ of the genes (Figure 1c). A full list of the OVA-induced genes is presented in supplementary Table 2 (http://www.jci.org/cgi/content/full/111/12/ 1863/DC1). Importantly, eotaxin-1 was reproducibly identified in the allergen-induced genes (Figure 1d). Quantitative analysis of the average difference signal for eotaxin-1 between allergen and saline revealed a 25 -fold induction $(P=0.001)$. Collectively, these data illustrated the potential value of the scientific approach; thus, providing the impetus for the next set of experiments. We aimed to determine the degree of overlap between allergen-induced global transcript profiles in two independent models of asthma. We analyzed experimental asthma induced by A. fumigatus antigen because this model involves a unique mucosal sensitization route (intranasal) compared with the OVA model (31) and because A. fumigatus is a ubiquitous and common aeroallergen. Importantly, both asthma models have similar phenotypes, including Th2-associated eosinophilic inflammation, mucus production, and airway hyperresponsiveness (AHR) (14-16). Lung RNA was obtained 18 hours after nine doses of intranasal A. fumigatus allergen or saline challenges (delivered over the course of 3 weeks). We compared allergen-challenged mice (OVA or Aspergillus) with their respective saline control mice, and genes were identified that statistically increased $(P<0.05)$ at least twofold after allergen challenge. Compared with mice challenged with saline, OVA-challenged mice had 496 genes induced and $A$. fumigatus-challenged mice had 527 genes induced (Figure 1e). A full list of A. fumigatus antigen-induced genes and the overlap with the OVA-induced genes are presented in supplementary Tables 3 and 4, respectively (http://www.jci.org/cgi/content/full/111/12/1863/ DC1). Notably, the majority (59\% of OVA and $55 \%$ of Aspergillus) of the induced transcripts overlapped between the two experimental asthma models (Figure 1e); however, there was a large subset of the identified genome specific to each model, indicating that although experimental asthma models have similar apparent phenotypes, there is large genetic diversity, as 
assessed by transcript profiles. This has important implications in understanding the diverse spectrum of genes potentially involved in human asthma.

Experimental asthma is associated with induction of genes involved in L-arginine metabolism. Our results identified a set of 291 genes that were commonly involved in disease pathogenesis rather than unique to a particular allergen or mode of disease induction (Figure 1e). These "asthma signature" genes provide a valuable opportunity to define new pathways involved in the pathogenesis of allergic airway inflammation. In this regard, we were struck by the high level of transcripts for genes involved in metabolism of L-arginine. Arginase I, arginase II, and the L-arginine transporter, cationic amino acid transporter (CAT)2, were strongly induced (Figure 2, a-c). We were particularly surprised to find induction of genes previously thought to be primarily involved in metabolic pathways in the liver. Other enzymes involved in L-arginine metabolism, such as argininosuccinate synthetase (AS), L-ornithine decarboxylase (L-ODC), and L-ornithine aminotransferase (L-OAT) were not significantly different between saline- and allergen-challenged mice (Figure $2 \mathrm{~d}$ and data not shown). Interestingly, microarray analysis revealed very specific dysregulation of arginase compared with NOS. For example, the hybridization signals for eNOS and neuronal NOS were below background in the saline- and allergen- challenged lung (data not shown). Although the iNOS mRNA was detectable under most conditions, it did not change significantly between saline and allergen challenge (Figure $2 \mathrm{e}$ and data not shown).

The enzyme arginase is a urea cycle enzyme that exists in two isoforms ( $60 \%$ amino acid homology), designated type I and type II, which are encoded by different genes on distinct chromosomes (12). Arginase I is a cytoplasmic protein that is primarily expressed in the liver; whereas arginase II is a mitochondrial protein expressed in a variety of tissues, especially the kidney and prostate (32). The exact function of arginase in extrahepatic tissue is not well understood. However, the product of arginase, L-ornithine, is a precursor in the production of polyamines (e.g., putrescine, spermidine, and spermine) and proline, which control cell proliferation and collagen production, respectively (Figure 2d) (12, 32). In fact, increased expression of arginase I alone is sufficient to increase the proliferation rates of vascular smooth muscle (24) and endothelial cells (25). Thus, arginase activity is potentially critically linked to cell growth and connective tissue production. Notably, both of these processes are key parameters in the pathogenesis of asthma (33). We subsequently determined by Northern blot analysis that there was a time- and dose-dependent induction of arginase I during the progression of OVA-induced experimental asthma; arginase I was induced 18 hours a

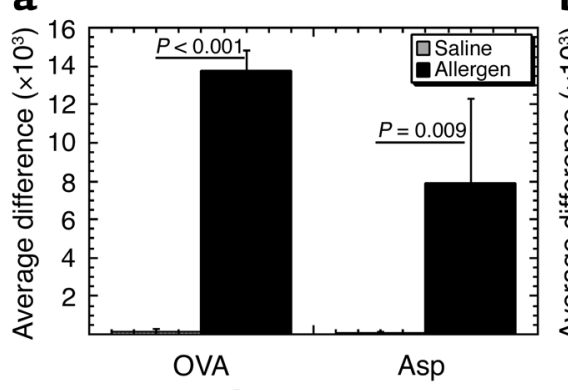

d

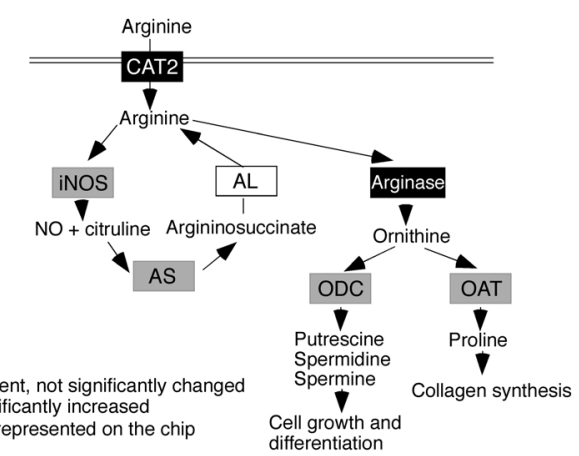

b
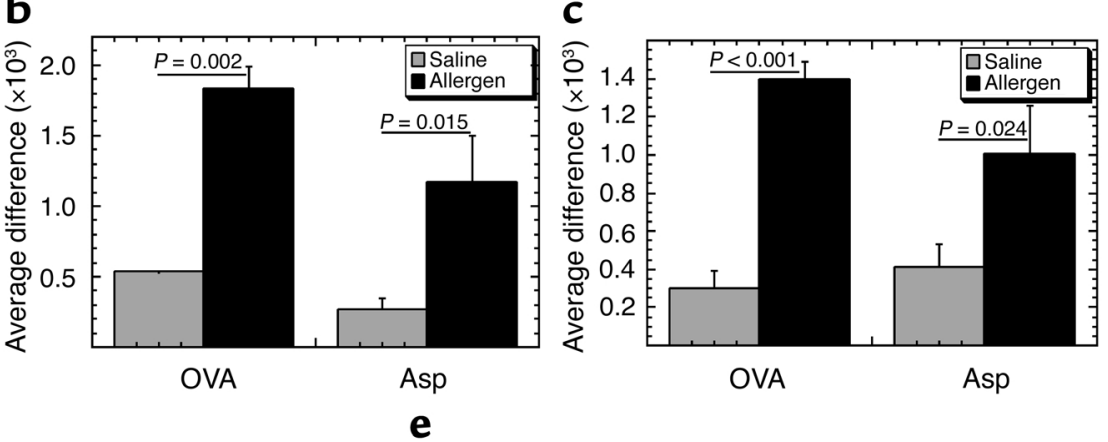

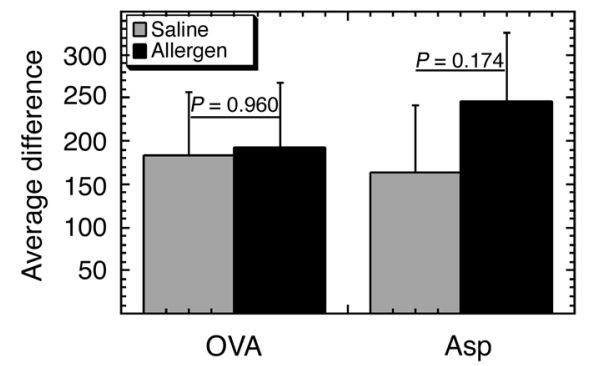

Figure 2

Expression of L-arginine metabolizing enzymes. Expression of arginase I (a), arginase II (b), CAT2 (c), and iNOS (e) in ovalbumin (OVA) and A. fumigatus (Asp)-challenged mice as measured by gene chip analysis is shown. The average difference for the hybridization signal after saline (gray bar) and allergen (black bar) challenge is depicted ( $n=2$ for Aspergillus control group and $n=3$ for OVA control group and OVA and Aspergillus experimental groups). Error bars represent the SD. A schematic representation of the L-arginine metabolism pathway is shown in d. Genes not present on the chip are depicted with a white box, genes present but not significantly increased with a gray box, and significantly increased genes with a black box. Abbreviations are AL, argininosuccinate lyase; AS, argininosuccinate synthetase; ODC, ornithine decarboxylase; and OAT, ornithine aminotransferase. 
a

\begin{tabular}{|c|c|c|c|c|c|}
\hline \multicolumn{2}{|c|}{$3 \mathrm{H}$} & \multicolumn{2}{|c|}{$18 \mathrm{H}$} & \multicolumn{2}{|c|}{$2 \mathrm{C}$} \\
\hline Sal & OVA & Sal & OVA & Sal & $\overline{\text { OVA }}$ \\
\hline
\end{tabular}
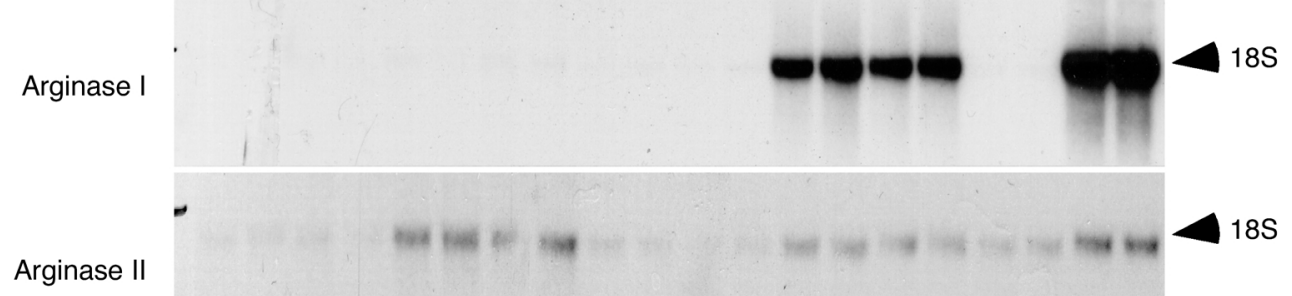

$\mathrm{EtBr}$

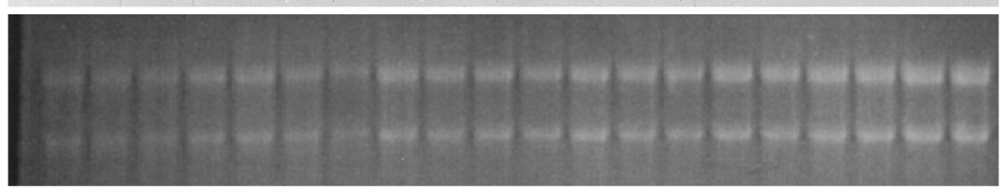

b

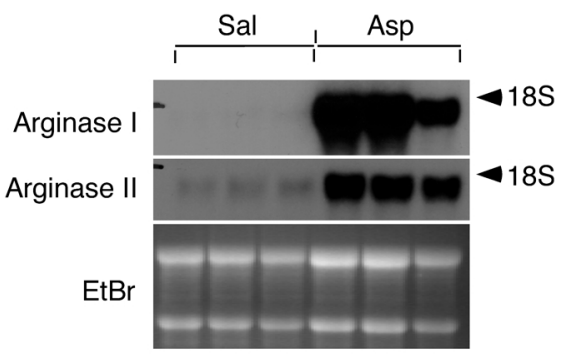

c

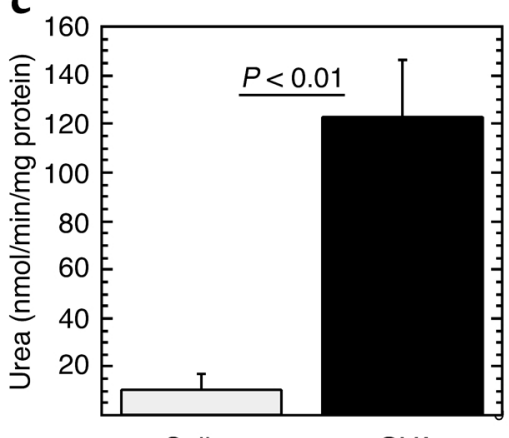

d

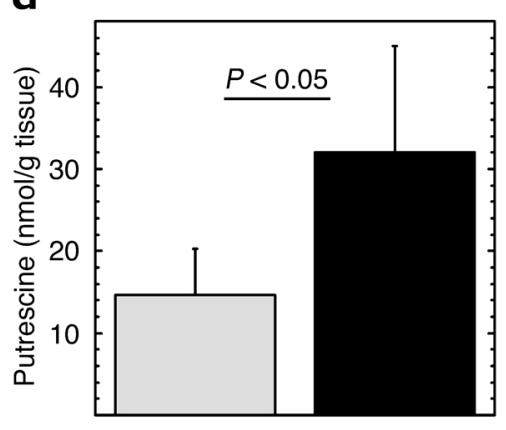

Saline

OVA

\section{Figure 3}

Northern blot and arginase activity analysis. In a, Northern blot analysis of arginase I and arginase II expression after OVA challenge is shown. Time points are as follows: $3 \mathrm{H}=1$ challenge, 3 hours; $18 \mathrm{H}=1$ challenge, 18 hours; $2 \mathrm{C}=2$ challenges, 18 hours. The EtBr-stained gel is also shown. The autoradiograph exposure times were 18 hours and 2 days for arginase I and arginase II, respectively. In $\mathbf{b}$, the expression of arginase I and arginase II after intranasal challenges with A. fumigatus or saline is shown. Sal, saline; Asp, aspergillus. The autoradiograph exposure times were 18 hours and 6 days for arginase I and arginase II, respectively. In $\mathbf{a}$ and $\mathbf{b}$, each lane represents a separate mouse. In $\mathbf{c}$, arginase activity in the lungs of saline- and OVA-challenged mice ( $n=4$ mice and $n=3$ mice, respectively) is shown. Arginase activity was measured in lung lysates with the use of the blood urea nitrogen reagent. As a control, arginase activity in the liver was $1522 \pm 183 \mathrm{and} 1390 \pm 78 \mathrm{nmol} / \mathrm{min} / \mathrm{mg} \mathrm{protein}$ for saline- and OVA-challenged mice, respectively. In d, putrescine levels in the whole lungs of saline- and OVA-challenged mice $(n=4$ mice and $n$ $=7$ mice, respectively) are shown. Putrescine levels were determined by HPLC.

after the first allergen challenge and even higher after two allergen challenges (Figure 3a). In addition, although arginase II mRNA induction was weaker than arginase I, it was induced earlier in the evolution of experimental asthma (Figure 3a). For example, arginase II was readily detectable 3 hours after the first allergen challenge (Figure 3a). The iNOS mRNA was weakly detectable and was not significantly induced by OVA challenge (data not shown). In addition, compared with mice challenged with nine doses of intranasal saline, A. fumigatus-challenged mice had marked expression of arginase I and arginase II (Figure 3b). Consistent with the results in the OVA model, there were only low levels of induction of iNOS mRNA (data not shown). Thus, the induction of arginase and CAT2 by allergen challenge was not specific to the antigen used but appeared to be part of the genetic program of experimental asthma. We hypothesized that the large increase in arginase mRNA would be translated into enhanced arginase activity in the lung because the activity of arginase is primarily regulated by a transcriptional mechanism (12). Indeed, after induction of OVA-induced experimental asthma, there was a marked increase in lung arginase activity (Figure 3c). Similar results were obtained when asthma was induced with A. fumigatus antigen; arginase activity was $10.8 \pm 10.8$ and $357 \pm 156 \mathrm{nmol} / \mathrm{min} / \mathrm{mg}$ protein ( $n=3-4$ mice; $P=0.02)$ for saline- and A. fumigatus-challenged mice, respectively. Consistent with the absence of arginase mRNA in the lung of control mice, the level of arginase activity in the saline-challenged lung was close to background. As a control, arginase activity in the liver was $1522 \pm 183$ and $1390 \pm 78 \mathrm{nmol} / \mathrm{min} / \mathrm{mg}$ protein for saline- and OVA-challenged mice, respectively. Finally, in the absence of OVA/alum sensitization, two doses of intranasal OVA did not induce 
arginase activity $(21.4 \pm 3.4$ and $26.2 \pm 4.4 \mathrm{nmol} /$ $\mathrm{min} / \mathrm{mg}$ protein $[n=4$ mice; $P>0.1]$ after saline and OVA, respectively), consistent with the requirement of an adaptive immune response for arginase induction rather than a primary innate induction mechanism (34). Although co-delivery of OVA with different doses of LPS can have profound effects on OVA-induced experimental asthma (34), our OVA preparation had undetectable levels of LPS.

Our results led us to the view that allergic responses are associated with marked induction of arginine metabolism via arginase. We were interested in proving that products downstream from arginase were actually overproduced in the allergic lung. Recent studies have shown that arginase is the rate-limiting enzyme for biosynthesis of putrescine, and that arginase expression can inhibit cellular apoptosis (35). We therefore examined putrescine levels in the lungs of asthmatic and control mice. Examining the whole lung, we determined that OVA-challenged mice had significantly increased levels of putrescine $(14.7 \pm 5.6$ vs. $32 \pm 13 \mathrm{nmol} / \mathrm{g}$ tissue $[P<0.05]$ in saline and OVA, respectively, Figure $3 \mathrm{~d}$ ). The twofold increase in putrescine is remarkable because we are examining whole lung levels of a mediator with distinct subcellular localization in the lung (36).

To begin to address the cellular sources of these molecules, we performed in situ hybridization for arginase I mRNA. Antisense staining of asthmatic lung (Figure 4, a and b, and data not shown) revealed high levels of arginase I in the perivascular and peribronchial pockets of inflammation. No specific staining with the sense probe in OVA-challenged mice was seen (Figure 4c). Hybridization of the antisense and sense probes in the saline-challenged lung was comparable with background (Figure $4 \mathrm{~d}$ and data not shown). There was a specific staining of the antisense probe to a subpopulation of large mononuclear cells with abundant cytoplasm (within the inflammatory infiltrates), most consistent with macrophages (Figure 4 , e and $\mathrm{f}$, and data not shown). In addition to analyzing the cellular distribution of arginase I $\mathrm{mRNA}^{+}$cells in the asthmatic lung, we were also interested in examining arginase expression by immunohistochemistry. Anti-arginase I immunohistochemistry revealed a very similar pattern of staining compared with the in situ hybridization studies. Specifically, the majority of staining was present in peribronchial and perivascular pockets of inflammation. Cells with the typical morphology of alveolar macrophages stained positive for arginase I protein (Figure 4g). Notably, saline control animals had undetectable arginase protein expression (data not shown). Finally, staining of allergen-challenged lungs with the macrophage marker Mac-3 revealed a similar pattern to arginase I expression (data not shown).

Collectively, these results challenge current paradigms about arginine metabolism in asthmatic responses that have previously focused on production of NO (37). We propose that arginine is metabolized by arginase, at least in part, in the asthmatic mouse lung. Furthermore, the variable levels of NO seen in asthma may be an indirect manifestation of arginase activity, an enzyme that functionally inhibits NOS by substrate depletion $(12,13)$. This model is consistent with the caution that has been advised with analysis of expired NO measurements in asthmatics (38).

Lung arginase is regulated by Th2 cytokines. Because asthma is a Th2-associated process and because IL- 4 has

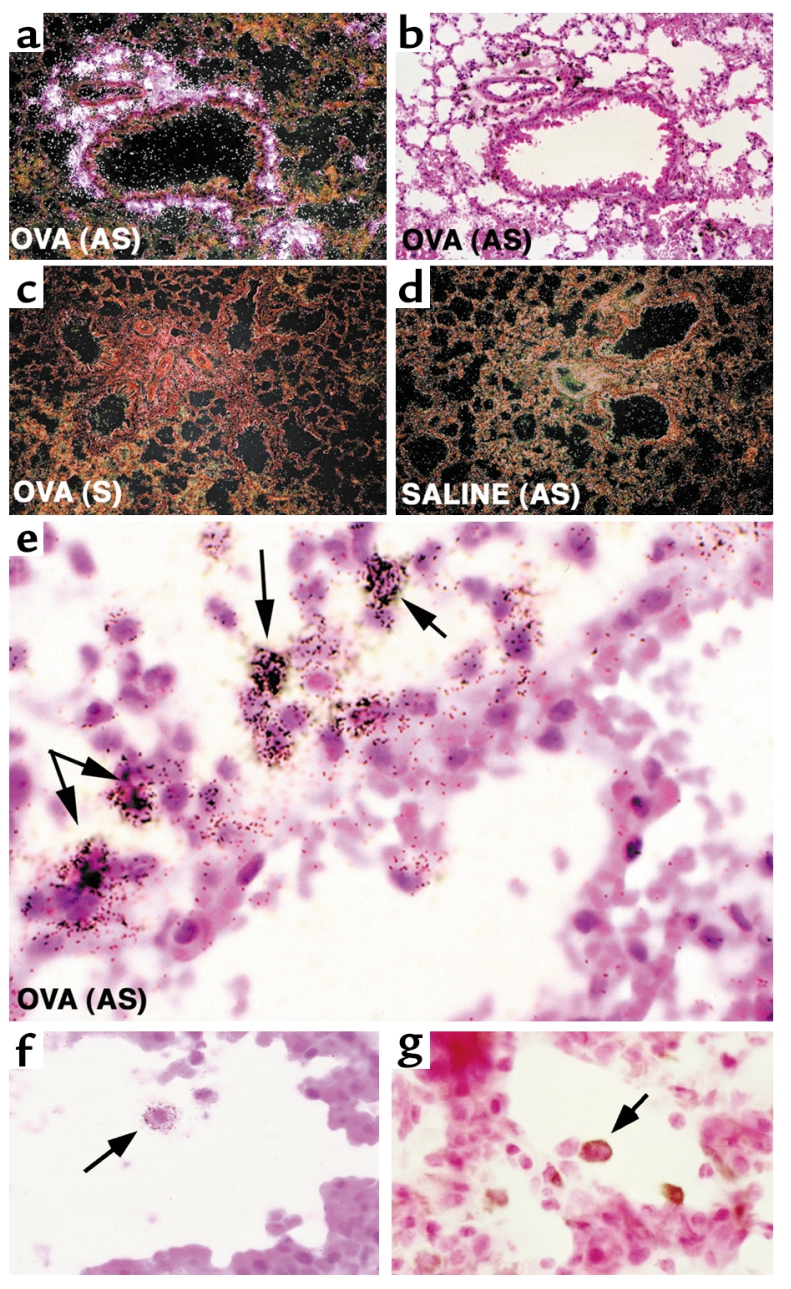

\section{Figure 4}

Arginase I mRNA in situ hybridization. The hybridization signal of the arginase I antisense (AS) and sense (S) probes are shown for OVA/alum sensitized mice challenged with two doses of OVA (a-c, e) or saline (d). Tissue was analyzed 18 hours after the second saline or allergen challenge. Bright field (b, e, f) and dark field images (a, c, d) are shown at $100 \times(\mathbf{a}-\mathbf{d})$ and $400 \times(\mathbf{e}, \mathbf{f})$ original magnification. The dark field signal is white/pink and the bright field signal is black. In the paired dark and bright field photomicrographs ( $\mathbf{a}$ and $\mathbf{b}$ ), a peribronchial staining pattern is shown. The hybridization of the AS probe to a subpopulation of isolated inflammatory cells is shown in e. Staining was also observed in isolated large mononuclear cells with abundant cytoplasm, typical for airway macrophages. Examples of such cells stained by in situ ( $\mathbf{f})$ and immunohistochemistry against arginase I (g) are shown. Arrows indicate representative positive signal. Representative photomicrographs of four separate mice are shown. 
a
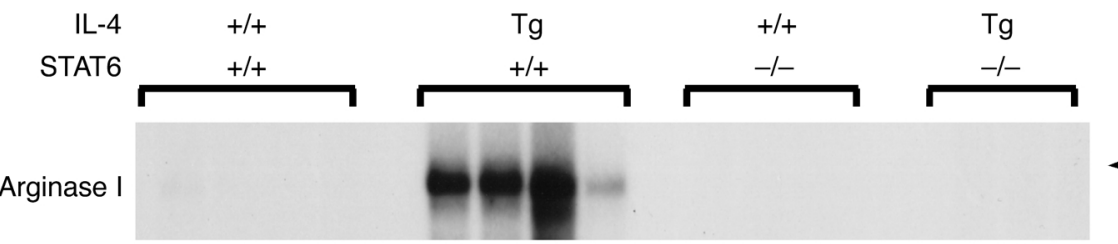

Arginase II
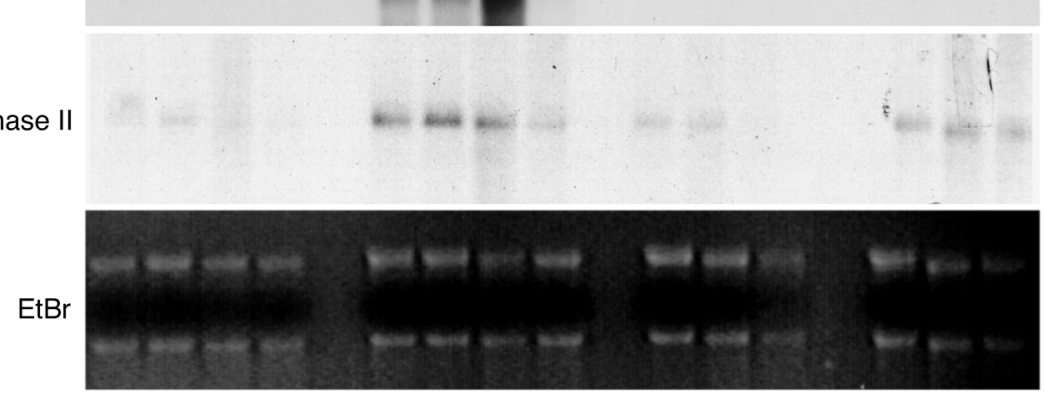

b

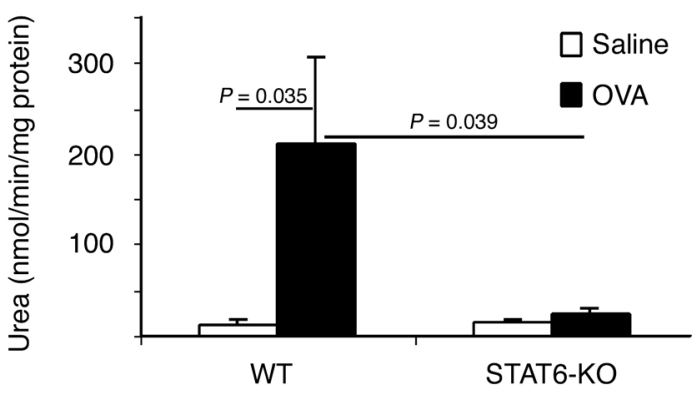

C

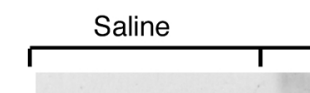

Arginase I

Arginase II

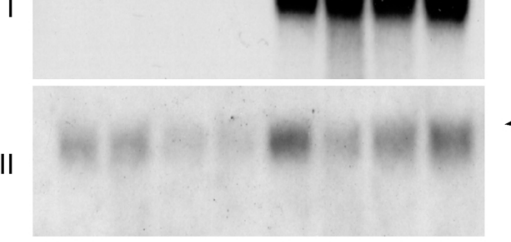

IL-13

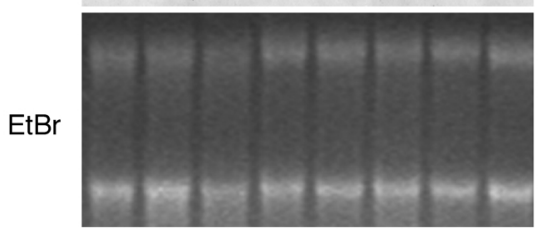

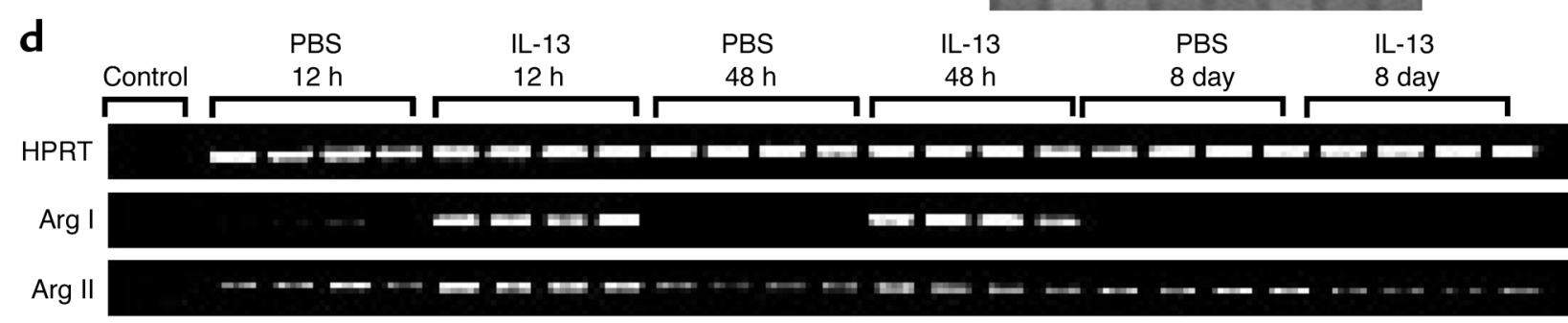

Figure 5

Regulation of arginase by IL-4, IL-13, and STAT6. Northern blot analyses of arginase I and arginase II in IL-4 lung transgenic mice (in the Balb/c background) containing either the wild-type or deleted STAT6 gene (a) and lungs from Balb/c mice treated with IL-13 intranasally (c) are shown. The position of the 18S RNA is shown. Each lane represents a separate mouse. EtBr staining of the RNA gels is also shown. In $\mathbf{b}$, arginase activity in the lungs of saline- and OVA-challenged ( $n=4$ and $n=3$, respectively) wild-type (WT) and STAT6-deficient (STAT6-KO, $n=4$ each) mice is shown. Arginase activity was measured in lung lysates with the use of the blood urea nitrogen reagent. In d, a kinetic characterization of IL-13 induced arginase mRNA levels in the lung is shown. Mice $(n=4-10$ per group) received one dose of intratracheal IL-13 (10 $\mu \mathrm{g})$ or PBS. Lung RNA was converted to CDNA and used for PCR analysis of arginase I (Arg I), arginase II (Arg II), or control hypoxanthine phosphoribosyltransferase (HPRT). The lane labeled "control" does not contain cDNA template.

been shown to induce arginase in several cell lines in vitro (e.g., macrophages, smooth muscle cells) $(23,39)$, we were interested in testing the hypothesis that overexpression of IL-4, particularly in the lungs, was sufficient for induction of arginase. Mice that overexpress the IL-4 transgene in pulmonary epithelium (under the control of the Clara cell 10 promoter) have several features of asthma, including eosinophil-rich inflammatory cell infiltrates, mucus production, changes in baseline airway tone (18), and possibly AHR (data not shown). We hypothesized that arginase mRNA would be induced by the IL-4 transgene. Indeed, IL-4 lung transgenic mice had a marked increase in the level of both arginase isoforms (Figure 5a).

IL-4 and IL-13 share similar signaling requirements such as utilization of the IL-4R $\alpha$ chain and the induction of janus kinase 1 and STAT6. A subset of their responses has been shown to be STAT6 dependent (19, 40). To test the role of STAT6 in the induction of arginase I in vivo, we examined IL-4 lung transgenic 
mice that contained wild-type or gene-targeted STAT6. As shown in Figure 5a, whereas IL-4 lung transgenic mice contained abundant arginase I mRNA, in the absence of STAT6, there was a complete loss of the IL-4-induced arginase I mRNA. Interestingly, the IL-4 transgene-induced arginase II mRNA signal was only partially attenuated (if at all) in STAT6-deficient mice (Figure 5a), indicating that arginase II, in contrast to arginase I, was largely STAT6 independent. These findings support in vitro studies that have demonstrated shared and distinct signaling requirements for these two isoenzymes (12). We were next interested in determining whether allergen-induced arginase was dependent on STAT6. This would determine whether allergeninduced arginase was predominantly downstream from IL-4 and IL-13 signaling. Notably, mice deficient in STAT6 had a $90 \%$ reduction in OVA-induced lung arginase activity (Figure $5 \mathrm{~b}$ ), suggesting that arginase I was the predominant inducible isoform in the asthmatic lung. Northern blot analysis indicated that both OVA- and Aspergillus-induced arginase I mRNA were STAT6 dependent (data not shown). Taken together, these findings indicate that induction of arginase during allergic lung inflammation is largely downstream from IL-4, IL-13, and STAT6. These results are consistent with the recent finding that IL-4 and IL-13 inhibit NO production in macrophages by a STAT6-dependent pathway (41). Consistent with these findings, the murine arginase I promoter contains a single STAT6 site that is required for response to IL-4 (12).

We were next interested in determining whether lung arginase was also induced by IL-13, a cytokine that has been shown to be critically involved in the development of several features of asthma $(10,42)$, and to induce arginase in cell lines in vitro $(23,41)$. To test this hypothesis, we administered IL-13 by repeated intranasal application to anesthetized mice. This protocol induces several features of experimental asthma, including eosinophilic inflammation, chemokine induction, mucus production, and AHR $(21,42)$. IL-13 administration induced marked levels of arginase I compared with saline-treated control mice (Figure 5c). Consistent with the finding that IL-4 transgenic mice had elevated levels of arginase II mRNA, IL-13 also increased arginase II mRNA levels (Figure 5c). To further analyze the ability of IL-13 to induce arginase, we treated a murine macrophage cell line (J774A.1) with IL-13 in vitro. Indeed, IL-13 induced arginase activity in a dose-dependent (0.5-50 $\mathrm{ng} / \mathrm{mL}$ ) manner (data not shown).

We have previously reported that one dose of intratracheal IL-13 induces marked AHR within 12 hours. We were therefore interested in performing a kinetic analysis of IL-13 induction of arginase. Notably, after only one dose of IL-13, the mRNA for the type I isoform was found to be induced already by 12 hours (Figure 5d); the type II isoform was constitutively present and induced to a lesser extent. Induction of arginase was detectable when early AHR developed
(21). The early induction of arginase and AHR precedes leukocyte recruitment (21 and data not shown). On the basis of these results, we speculate that the induction of AHR by IL-13 may be related to the ability of arginase to functionally inhibit production of the bronchodilator NO by substrate depletion $(12,13)$.

Arginase is induced in buman asthma. We were interested in determining whether our findings in experimental asthma in mice were applicable to human asthma. To begin to translate our results into humans, we analyzed arginase I protein expression in bronchoalveolar lavage fluid cells from individuals with asthma and control patients. With the use of immunocytochemistry, there were a significantly higher number of cells expressing arginase I in the asthmatic group (Figure 6a). In both groups, the immunopositive cells were predominantly mononuclear cells with macrophage morphology. A small population of immunopositive granulocytes was present in the asthmatic group (data not shown).

Finally, we performed in situ hybridization on bronchial biopsy specimens from patients with asthma. Arginase I $\mathrm{mRNA}^{+}$cells were strongly detected in the asthmatic lung but were almost completely undetectable in control individuals $(n=4$ asthmatic and 4 control individuals). No signal was seen with the control sense probe (data not shown). Similar to the results with the mouse models, staining was observed in submucosal inflammatory cell infiltrates (Figure 6b). Interestingly, we also observed staining for arginase I mRNA in epithelial cells (Figure 6b).

\section{Discussion}

We have carried out DNA microarray profile analysis of mice undergoing experimental asthma. By using two established models of experimental asthma, we

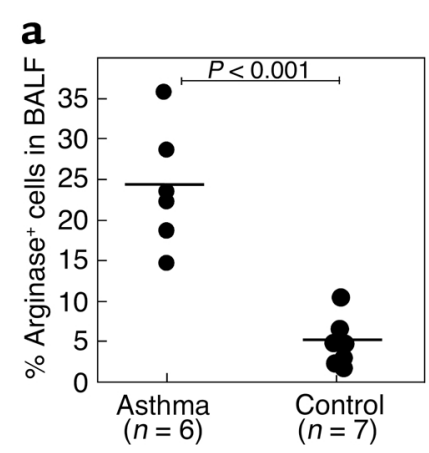

\section{b}

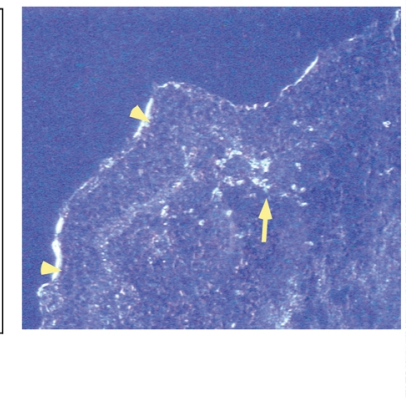

\section{Figure 6}

Arginase I protein expression in human asthma. Fiberoptic bronchoscopy of allergic asthmatics and healthy controls was conducted, and BALF was analyzed for arginase I by immunohistochemistry (a). The number of immunopositive cells, expressed as a percentage of total cells, is shown. In $\mathbf{b}$, a representative dark field illumination (blue filter, 200x original magnification) of arginase I mRNA in situ hybridization of cryostat section from an asthmatic biopsy specimen by using a $\mathrm{S}^{35}$-labeled RNA probe is shown. Signal was detected mostly in the inflammatory cells in the mucosa (arrow). Patchy mRNA positive cells were also detected within the epithelium (arrowheads). These results are representative of four asthmatic individuals. 
identified genes that were significantly dysregulated in the allergic response. Our data show that $6.5 \%$ of the tested genome was dysregulated during induction of experimental asthma. We demonstrate that the majority of induced genes were similar between the two allergen-challenged models, implicating common pathways and allowing us to define a set of asthma signature genes. At the same time, we report that a large subset of the identified genome is specific to each experimental model, indicating significant genetic diversity despite similar apparent asthma phenotypes. These data have significant medical implications because they suggest that clinically similar patients may have large differences in molecular pathogenesis of their individual disease. As such, DNA transcript profiling may eventually provide greater predictive value than present phenotypic markers (e.g., degree of eosinophilia, AHR, or histopathology).

We were struck by the finding that the gene for arginase I was reproducibly present among the allergen-induced genes. In addition, the genes for arginase II and the L-arginine transporter, CAT2, were increased, providing further evidence for an important role for arginine metabolism in allergic airway responses. On the basis of the potential importance of L-arginine metabolism via arginase in the pathogenesis of asthma, and the association of arginase with in vitro Th2 responses, we focused our attention on the regulation and role of arginase in experimental asthma. In situ mRNA hybridization analysis of the lung from asthmatic mice revealed marked induction of arginase I mRNA in the peribronchial and perivascular inflammatory sites with staining in multiple cell types, especially macrophages. In addition, arginase expression in the lung was induced by IL-4 and IL-13 in a STAT6dependent manner. Although numerous factors may influence gene expression (e.g., technical procedures, adjuvants, and routes of antigen administration), our data indicate that the arginase pathway is not dependent on these factors because arginase is induced by both asthma models and by Th2 cytokine overexpression (by pharmacologic and transgenic delivery). These findings are supported by the recent observation that NOS and arginase are differentially regulated during Th1- and Th2-associated granulomatous responses in mice, respectively (43). Finally, we demonstrate that arginase is induced in human asthma supporting the importance of this pathway in human disease. Interestingly, in situ hybridization in the human asthmatic lung revealed expression in submucosal inflammatory cells and also airway epithelium, further extending our findings from the mouse. Notably, airway epithelium is a major location of arginine and polyamines in the asthmatic lung $(36,44)$.

We propose that arginase induction by IL-4/IL-13 signaling is not just a marker of allergic airway responses, but that arginase is involved in the pathogenesis of multiple aspects of asthma. Previous studies have demonstrated the involvement of arginine metabolism pathways in asthma, but these studies have primarily focused on metabolism by NOS. Our study is the first to demonstrate the involvement of arginase and the arginine transporter, CAT2. Our demonstration that CAT2 is overexpressed is consistent with reports of the elevated levels of arginine and nitrotyrosine in airway epithelial cells of asthmatic subjects $(44,45)$. Previous studies have shown that pharmacologic inhibition of NOS enhances agonistinduced airway constriction in vitro $(46,47)$ and in vivo $(46,48,49)$. In addition, in a guinea pig model of asthma, a specific deficiency of bronchodilating cNOS-derived NO has been shown to contribute to AHR $(47,48,50)$. It has been suggested that the NO deficiency associated with experimental asthma is attributable, at least in part, to the ability of eosinophil cationic proteins to inhibit L-arginine uptake by macrophages (51). Our current findings suggest that the NO deficiency associated with asthma may be largely mediated by arginase induction. By regulating the availability of L-arginine for NOS (12), arginase induction may have an important impact on the induction of AHR. It is interesting to note that IL-13-induced AHR occurs in a time frame that parallels arginase induction, suggesting that the ability of IL-13 to promote AHR may be directly dependent on arginase induction. This may be particularly important because IL-13 has been recently shown to be a major effector cytokine in asthma pathogenesis (10, 42). Notably, the mechanism by which IL-13 induces AHR has been unclear; our results draw attention to the ability of IL-13 to regulate arginase as a possible mechanism of AHR. In addition to affecting AHR, we propose that arginase downstream products (proline and polyamines) are also involved in asthma pathogenesis. Indeed, we have found elevated levels of putrescine in the asthmatic murine lung, and elevated levels of polyamines have been reported in the serum of patients with asthma (52), corroborating the production of biologically active mediators downstream from arginase. Interestingly, polyamines have contractile activity on smooth muscle $(53,54)$, possibly implicating them in asthma-associated AHR. Polyamines are present in multiple cell types in the lung (including airway epithelium, smooth muscle cells, and macrophages) (36). The ability of polyamines to affect multiple processes, including cell survival, cell proliferation, and mucus production $(55,56)$, indicates that they may have numerous functions in several cell types present in the asthmatic lung.

Work from several laboratories has led to the notion that macrophage-derived arginase has a role in the recovery of host tissues from inflammation (13). This effect is not only mediated by competing with NOS for L-arginine as a substrate, but also by generating L-ornithine for synthesis of proline (12). This may be particularly important in inflammatory sites characterized by tissue remodeling/repair (such as the asthmatic airway) because proline often becomes a rate 
limiting substrate for collagen synthesis $(57,58)$. Under these conditions, it is believed that extracellular L-ornithine and proline, secreted from arginase expressing cells (e.g., macrophages) are transported into fibroblasts, where they subsequently become incorporated into collagen $(57,58)$.

In summary, we have described a pathway (involving arginine metabolism by arginase) not previously examined in the context of allergic airway inflammation. Our results challenge the conventional view that arginine is primarily metabolized by NOS in asthmatic responses; rather, we propose that significant metabolism occurs by arginase, and that this process has important ramifications on the manifestations of disease. As such, this new pathway may represent an important therapeutic intervention strategy for the treatment of allergic lung disease.

\section{Acknowledgments}

We thank Andrea Lippelman for editorial assistance and Fred Finkelman for helpful discussions and critical review of this manuscript. We are grateful to Sidney Morris for the RG-1 antibody, Christopher Karp for performing the LPS measurements, and to Debra Donaldson for IL-13. This work was supported in part by the American Heart Association Scientist Development (N. Zimmermann) and Post-doctorate fellowship (N. King) grants; NIH grants R01 AI42242-05, AI45898-04, and AI53479-01 (all to M.E. Rothenberg); the Human Frontier Science Program (M.E. Rothenberg, P.S. Foster); International Life Sciences Institute (M.E. Rothenberg); and Burroughs Wellcome Fund (M.E. Rothenberg).

1. Holgate, S.T. 1999. The epidemic of allergy and asthma. Nature. 402:B2-B4.

2. Umetsu, D.T., McIntire, J.J., Akbari, O., Macaubas, C., and DeKruyff, R.H. 2002. Asthma: an epidemic of dysregulated immunity. Nat. Immunol. 3:715-720.

3. Broide, D.H. 2001. Molecular and cellular mechanisms of allergic disease. J. Allergy Clin. Immunol. 108:S65-S71.

4. Busse, W.W., and Lemanske, R.F., Jr. 2001. Asthma. N. Engl. J. Med. 344:350-362.

5. Lee, N.A., Gelfand, E.W., and Lee, J.J. 2001. Pulmonary T cells and eosinophils: coconspirators or independent triggers of allergic respiratory pathology? J. Allergy Clin. Immunol. 107:945-957.

6. Robinson, D.S., et al. 1992. Predominant TH2-like bronchoalveolar T-lymphocyte population in atopic asthma. N. Engl. J. Med. 326:298-304

7. Drazen, J.M., Arm, J.P., and Austen, K.F. 1996. Sorting out the cytokines of asthma. J. Exp. Med. 183:1-5.

8. Ray, A., and Cohn, L. 1999. Th2 cells and GATA-3 in asthma: new insights into the regulation of airway inflammation. J. Clin. Invest. 104:985-993.

9. Bochner, B.S., Undem, B.J., and Lichtenstein, L.M. 1994. Immunological aspects of allergic asthma. Annu. Rev. Immunol. 12:295-335.

10. Wills-Karp, M. 2001. IL-12/IL-13 axis in allergic asthma. J. Allergy Clin. Immunol. 107:9-18.

11. Barnes, P.J. 2000. New directions in allergic diseases: mechanism-based anti-inflammatory therapies. J. Allergy Clin. Immunol. 106:5-16.

12. Morris, S.M., Jr. 2002. Regulation of enzymes of the urea cycle and arginine metabolism. Annu. Rev. Nutr. 22:87-105.

13. Mills, C.D. 2001. Macrophage arginine metabolism to ornithine/urea or nitric oxide/citrulline: a life or death issue. Crit. Rev. Immunol. 21:399-425

14. Mishra, A., Weaver, T.E., Beck, D.C., and Rothenberg, M.E. 2001. Interleukin-5-mediated allergic airway inflammation inhibits the human surfactant protein $\mathrm{C}$ promoter in transgenic mice. J. Biol. Chem. 276:8453-8459.

15. Mehlhop, P.D., et al. 1997. Allergen-induced bronchial hyperreactivity and eosinophilic inflammation occur in the absence of IgE in a mouse model of asthma. Proc. Natl. Acad. Sci. U. S. A. 94:1344-1349.

16. Kurup, V.P., Seymour, B.W., Choi, H., and Coffman, R.L. 1994. Particulate Aspergillus fumigatus antigens elicit a TH2 response in $\mathrm{BALB} / \mathrm{C}$ mice. J. Allergy Clin. Immunol. 93:1013-1020.

17.Zimmermann, N., et al. 2000. Murine eotaxin-2: a constitutive eosinophil chemokine induced by allergen challenge and IL- 4 overexpression. J. Immunol. 165:5839-5846.

18. Rankin, J.A., et al. 1996. Phenotypic and physiologic characterization of transgenic mice expressing interleukin 4 in the lung: lymphocytic and eosinophilic inflammation without airway hyperreactivity. Proc. Natl. Acad. Sci. U. S. A. 93:7821-7825.

19. Shimoda, K., et al. 1996. Lack of IL-4-induced Th2 response and IgE class switching in mice with disrupted Stat6 gene. Nature. 380:630-633.

20. Pope, S.M., et al. 2001. IL-13 induces eosinophil recruitment into the lung by an IL-5- and eotaxin-dependent mechanism. J. Allergy Clin. Immunol. 108:594-601.

21. Yang, M., et al. 2001. Interleukin-13 mediates airways hyperreactivity through the IL-4 receptor-alpha chain and STAT- 6 independently of IL-5 and eotaxin. Am. J. Respir. Cell Mol. Biol. 25:522-530.

22. Matthews, A.N., et al. 1998. Eotaxin is required for the baseline level of tissue eosinophils. Proc. Natl. Acad. Sci. U. S. A. 95:6273-6278.

23. Wei, L.H., Jacobs, A.T., Morris, S.M., Jr., and Ignarro, L.J. 2000. IL-4 and IL-13 upregulate arginase I expression by cAMP and JAK/STAT6 pathways in vascular smooth muscle cells. Am. J. Physiol. Cell Physiol. 279:C248-C256

24. Wei, L.H., Wu, G., Morris, S.M., Jr., and Ignarro, L.J. 2001. Elevated arginase I expression in rat aortic smooth muscle cells increases cell proliferation. Proc. Natl. Acad. Sci. U. S. A. 98:9260-9264.

25. Li, H., et al. 2002. Activities of arginase I and II are limiting for endothelial cell proliferation. Am. J. Physiol. Regul. Integr. Comp. Physiol. 282:R64-R69.

26. Lopatin, A.N., Shantz, L.M., Mackintosh, C.A., Nichols, C.G., and Pegg, A.E. 2000. Modulation of potassium channels in the hearts of transgenic and mutant mice with altered polyamine biosynthesis. J. Mol. Cell Cardiol. 32:2007-2024.

27. American Thoracic Society. 1987. Standards for the diagnosis and care of patients with chronic obstructive pulmonary disease (COPD) and asthma. Am. Rev. Respir. Dis. 136:225-244.

28. Olivenstein, R., Taha, R., Minshall, E.M., and Hamid, Q.A. 1999. IL-4 and IL-5 mRNA expression in induced sputum of asthmatic subjects: comparison with bronchial wash. J. Allergy Clin. Immunol. 103:238-245.

29. Hamid, Q. 1997. Immunohistochemistry. In Allergy and allergic disease. A.B. Kay, editor. Blackwell Science Ltd. London, United Kingdom. $775-778$.

30. Rothenberg, M.E., MacLean, J.A., Pearlman, E., Luster, A.D., and Leder, P. 1997. Targeted disruption of the chemokine eotaxin partially reduces antigen-induced tissue eosinophilia. J. Exp. Med. 185:785-790

31. Kurup, V.P., Mauze, S., Choi, H., Seymour, B.W., and Coffman, R.L. 1992. A murine model of allergic bronchopulmonary aspergillosis with elevated eosinophils and IgE. J. Immunol. 148:3783-3788.

32. Iyer, R., et al. 1998. The human arginases and arginase deficiency. J. Inherit. Metab. Dis. 21:86-100.

33. Elias, J.A., Zhu, Z., Chupp, G., and Homer, R.J. 1999. Airway remodeling in asthma. J. Clin. Invest. 104:1001-1006.

34. Eisenbarth, S.C., et al. 2002. Lipopolysaccharide-enhanced, toll-like receptor 4-dependent $\mathrm{T}$ helper cell type 2 responses to inhaled antigen. J. Exp. Med. 196:1645-1651.

35. Esch, F., et al. 1998. Purification of a multipotent antideath activity from bovine liver and its identification as arginase: nitric oxide-independent inhibition of neuronal apoptosis. J. Neurosci. 18:4083-4095.

36. Hoet, P.H., and Nemery, B. 2000. Polyamines in the lung: polyamine uptake and polyamine-linked pathological or toxicological conditions. Am. J. Physiol. Lung Cell Mol. Physiol. 278:L417-L433.

37. Kharitonov, S.A., and Barnes, P.J. 2000. Clinical aspects of exhaled nitric oxide. Eur. Respir. J. 16:781-792.

38. Hunt, J., and Gaston, B. 2000. Airway nitrogen oxide measurements in asthma and other pediatric respiratory diseases. J. Pediatr. 137:14-20.

39. Munder, M., et al. 1999. Th1/Th2-regulated expression of arginase isoforms in murine macrophages and dendritic cells. J. Immunol. 163:3771-3777.

40. Ihle, J.N. 2001. The Stat family in cytokine signaling. Curr. Opin. Cell Biol. 13:211-217.

41. Rutschman, R., et al. 2001. Cutting edge: Stat6-dependent substrate depletion regulates nitric oxide production. J. Immunol. 166:2173-2177.

42. Grunig, G., et al. 1998. Requirement for IL-13 independently of IL-4 in experimental asthma. Science. 282:2261-2263.

43. Hesse, M., et al. 2001. Differential regulation of nitric oxide synthase-2 and arginase- 1 by type 1/type 2 cytokines in vivo: granulomatous pathology is shaped by the pattern of L-arginine metabolism. J. Immunol. 167:6533-6544. 
44. Guo, F.H., et al. 2000. Molecular mechanisms of increased nitric oxide (NO) in asthma: evidence for transcriptional and post-translational regulation of NO synthesis. J. Immunol. 164:5970-5980.

45. MacPherson, J.C., et al. 2001. Eosinophils are a major source of nitric oxide-derived oxidants in severe asthma: characterization of pathways available to eosinophils for generating reactive nitrogen species. J. Immunol. 166:5763-5772.

46. Nijkamp, F.P., van der Linde, H.J., and Folkerts, G. 1993. Nitric oxide synthesis inhibitors induce airway hyperresponsiveness in the guinea pig in vivo and in vitro. Role of the epithelium. Am. Rev. Respir. Dis. 148:727-734.

47. De Boer, J., et al. 1996. Deficiency of nitric oxide in allergen-induced airway hyperreactivity to contractile agonists after the early asthmatic reaction: an ex vivo study. Br. J. Pharmacol. 119:1109-1116.

48. Schuiling, M., et al. 1998. Role of nitric oxide in the development and partial reversal of allergen-induced airway hyperreactivity in conscious, unrestrained guinea-pigs. Br. J. Pharmacol. 123:1450-1456.

49. Taylor, D.A., McGrath, J.L., Orr, L.M., Barnes, P.J., and O'Connor, B.J. 1998. Effect of endogenous nitric oxide inhibition on airway responsiveness to histamine and adenosine-5'-monophosphate in asthma. Thorax. 53:483-489.

50. Schuiling, M., Meurs, H., Zuidhof, A.B., Venema, N., and Zaagsma, J. 1998. Dual action of iNOS-derived nitric oxide in allergen-induced airway hyperreactivity in conscious, unrestrained guinea pigs. Am. J. Respir. Crit. Care Med. 158:1442-1449.

51. Hammermann, R., et al. 1999. Cationic proteins inhibit L-arginine uptake in rat alveolar macrophages and tracheal epithelial cells. Implications for nitric oxide synthesis. Am. J. Respir. Cell Mol. Biol. 21:155-162.

52. Kurosawa, M., Shimizu, Y., Tsukagoshi, H., and Ueki, M. 1992. Elevated levels of peripheral-blood, naturally occurring aliphatic polyamines in bronchial asthmatic patients with active symptoms. Allergy. 47:638-643.

53. Nilsson, B.O., and Hellstrand, P. 1993. Effects of polyamines on intracellular calcium and mechanical activity in smooth muscle of guineapig taenia coli. Acta. Physiol. Scand. 148:37-43.

54. Sward, K., Pato, M.D., Nilsson, B.O., Nordstrom, I., and Hellstrand, P. 1995. Polyamines inhibit myosin phosphatase and increase LC20 phosphorylation and force in smooth muscle. Am. J. Physiol. 269:C563-C571.

55. Thomas, T., and Thomas, T.J. 2001. Polyamines in cell growth and cell death: molecular mechanisms and therapeutic applications. Cell Mol. Life Sci. 58:244-258.

56. Ma, L., Wang, W.P., Chow, J.Y., Lam, S.K., and Cho, C.H. 2000. The role of polyamines in gastric mucus synthesis inhibited by cigarette smoke or its extract. Gut. 47:170-177.

57. Albina, J.E., Abate, J.A., and Mastrofrancesco, B. 1993. Role of ornithine as a proline precursor in healing wounds. J. Surg. Res. 55:97-102.

58. Kershenobich, D., Fierro, F.J., and Rojkind, M. 1970. The relationship between the free pool of proline and collagen content in human liver cirrhosis. J. Clin. Invest. 49:2246-2249. 